the contact time.

It is important that practitioners of hospital disinfection have this perspective when assessing reports such as that by Townsend et al. For example, their procedure consisted first of bottlebrush cleansing with detergent solution, followed by four water rinses and some air-drying. Their figures show that this physical disinfection step reduced the number of contaminated tubes from $92 \%$ to $72 \%$ and the mean survivor count by more than $99 \%$. Yet, the article's title puts great emphasis on the chemical component even indicating its composition. Although the authors did mention the "detergent wash" in the text, they also said, “... our study design did not permit us to determine if the cleansing procedure was necessary..." The implication is clear that the chemical might have done as much without pre-cleansing. The writer from long experience with tests of this nature can attest that that is most unlikely.

Another reason for questioning the emphasis on chemical action is that the data fail to provide the information needed for the chemical efficacy evaluation that is part of the title. To be able to do this there should have been a control in which water was substituted for disinfectant. Consequently the role of the chemical component has to be surmised from other results. Following contact with chemical (followed by three rinses and air-drying) there were significant reductions in the number of contaminated tubes, but the proportionate reduction in the mean count was of the same order as that following the detergent-wash alone. And among the $\mathbf{3 0}$ different types of survivors were some that are generally quite susceptible to disinfectants (Neisseria, for example). This finding leads one to suspect that the level of chemical action may not have been high.

There is a great need for in-use studies on equipment disinfection that provide a balanced assessment of the whole decontamination procedure and compare two or more procedures one of which is in common use. The purpose of this communication is to encourage such studies.

Earle H. Spaulding, Ph.D. Department of Microbiology School of Medicine Temple University Philadelphia, Pennsylvania
Dr. Timothy R. Townsend, who authored the article in question, was invited to respond.

Dr. Spaulding is correct in that to evaluate the specific contribution to the disinfection process of either the pre-cleansing procedure or the disinfectant itself, a different study design would be needed. It was not our intent to imply that pre-cleansing was not necessary. Our intent was quite the opposite, to caution the reader that our study design was such that the importance of pre-cleansing could not be properly evaluated.

I agree with Dr. Spaulding that there is a great need for more in-use studies that provide a balanced assessment of the entire decontamination procedure. Our hope was that our study might stimulate more and better studies. With Dr. Spaulding's permission, I would like to extend his plea for more studies to include controlled studies that directly compare different disinfectants available to hospitals. In this regard, it was very unfortunate that funding was withdrawn in 1981 for the Centers for Disease Control sponsored study (Microbiologic Evaluation of Chemicals and Methods Used for High-Level Disinfection of In-Use, Naturally Contaminated Respiratory Therapy Breathing Circuits, RFP No. 200-81-0628) which would have evaluated both manual and machine processing of ventilator tubing as well as many different types of disinfectants. Such a study would have been invaluable not only in providing practical inuse data to hospitals, allowing them to choose the best disinfectant for the job, but in furthering our understanding of which components of the disinfection process are most efficacious.

Timothy R. Townsend, M.D. Assistant Professor of Pediatrics Hospital Epidemiologist

The Johns Hopkins Hospital Baltimore, Maryland

\section{Length of Sterility in Self Sealing Wrap}

\section{To the Editor:}

Regarding the article in Infection Control, Vol. 2, No. 2, page 143, we use "Ameri-Wrap Self Sealing Wrap" of American Hospital Supply Corporation for gas and heat sterilization.
Have you done studies on length of sterility in this type of a wrap? At this time we allow six months sterility in a slosed cabinet for the wrap.

Bobbi Bachelder, R.N. Infection Control Nurse Long Prairie Memorial Hospital \& Home Long Prairie, Minnesota

The preceding letter was referred to George F. Mallison, MPH, PE, for a reply.

I have seen no articles in medical or scientific literature on studies of the safe length of sterility using this particular product. However, the wrapper appears to be essentially the same as a number of other wrappers it consists of transparent plastic (probably polypropylene or $\mathrm{PVC}$ ) on one side bonded on the other to white kraft paper. Sold as a pouch, it has a presson seal (that appears quite effective) on one end and easy-to-open tabs on the other end.

Studies on the safe storage times of pouch-type wrappers were reported by Dineen (AORN Journal 13:63-64, 1971). His work indicated that sterile storage for more than one year was possible.* Nonetheless it seems entirely inappropriate to me to keep in storage any sterile-wrapped item more than a few weeks: long-term storage represents an expensive, unused inventory. I recommend a considerably shorter time for safe use, to reduce both inventories as well as the chance of excessive handling of pouches causing cuts or tears in packaging. Three months would seem to be reasonable.

George F. Mallison, MPH, PE Consultant, Environmental \& Infection Control Glen Rock, New Jersey

\footnotetext{
- The 1981 CDC Guidelines for Hospital Environmental Control recommended that such types of wrappers (if heat sealed and, as indicated above, the press-on seal appears effective) should provide sterility for at least one year (Infect Control 1981; 2:143.).
} 


\section{Coming soon...}

\section{The Ultimate Drainage System for just s:124:5! \\ Hide-Away ${ }^{\mathrm{T}}$}

twin hangers pop-up

when you need them

to keep bag level

on windy days.

Dial-a-Drip
valve lets you calibrate drip rates.

A sumprise to the patient; a convenience for the nurse.

Flexible Armor-Tuf ${ }^{\mathrm{T}}$ tubing rated to withstand 1200 psi.

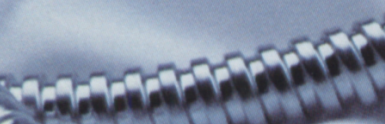

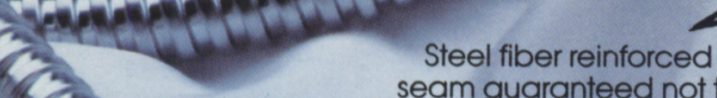

Steel fiber reinforced
seam guaranteed not to

tear for three years.

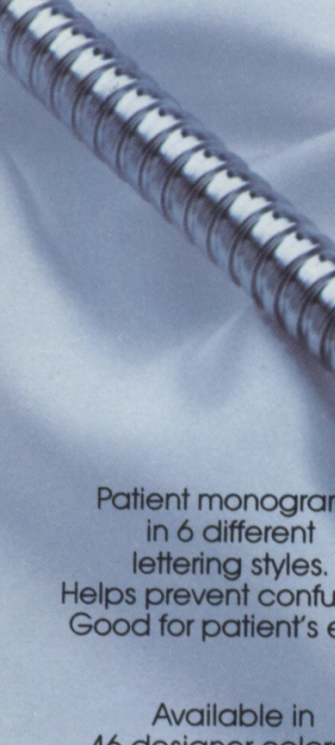

16 designer colors to match any room decor.
Non-corroding

stainless steel exit port with No-Drip ${ }^{\text {TM }}$ shut-off valve.

Built-in Accu-Scale ${ }^{\mathrm{TM}}$ displays bag weight to the nearest gram.

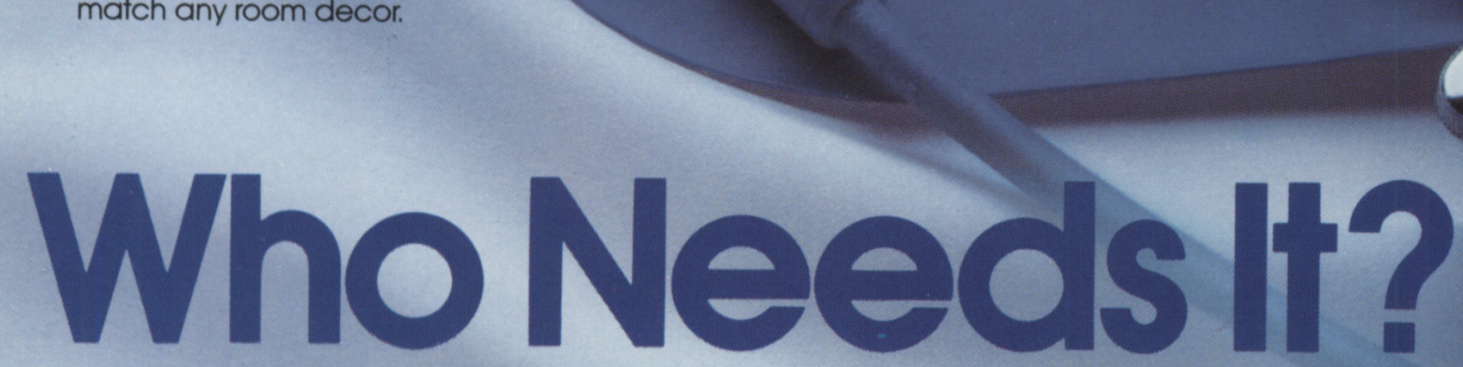




\section{Seamless already gives you what you really need.}

All urinary drainage systems perform the same functions. So why pay a jacked-up price for frills when all you really need is a Seamless system that satisfies all these needs: (1) ensures closed system integrity; (2) minimizes the risks of infection; (3) ensures unobstructed catheter drainage; (4) bags empty easily, completely; (5) saves nursing time.

Seamless satisfies CDC recommendations

You won't pay for expensive "bells and whistles" with Seamless. Yet, you'll have everything you need to satisfy the CDC recommendations as outlined in "Guidelines for Prevention of Catheter-Associated Urinary Tract Infections

and Guideline Ranking Scheme." Here's what the CDC

has to say about all the so-called "improvements" manufacturers have been touting for years"... none have been shown to be effective in reducing the frequency of catheter-associated infections. Additionaly, overly complex drainage systems can affect the ease of operation or more easily malfunction."

Patient comfort and nursing convenience built in

Seamless offers what's needed to make patients more comfortable and the nurse's job easier.

Vented drainage systems provide a free flow of urine for maximum drainage and ball-check anti-reflux valves help prevent retrograde contamination without obstructing flow.

Resealable sample ports let the nurse easily draw urine samples without violating closed system integrity. Large graduations are easy-

to-read from a distance. And all Seamless bags are easy to hang and drain ... quickly. completely. Latex bottom drain is packaged closed to insure the sterile integrity of the system.

A complete line to meet your needs

To save you money, Seamless offers variety so you can select the features you need only when you need them. Seamless also offers an extensive line of easy-to-use catheter trays for both indwelling and intermiltent calheters, drainage bags, urine meters, and sterile irigation systems.

All you give up is a high price Why pay for frills? Seamless gives you everything you really need to ensure patient comfort, p'sising convenience and protection ac a ainst sinary tract infections.

*Kunin CM, McCormack RC. Prevention of catheterinduced urinary tract infections by sterile closed

drainage. N Engl J Med 1966; 274: 1155-61, as quoted in CDC Guidelines, October, 1981.

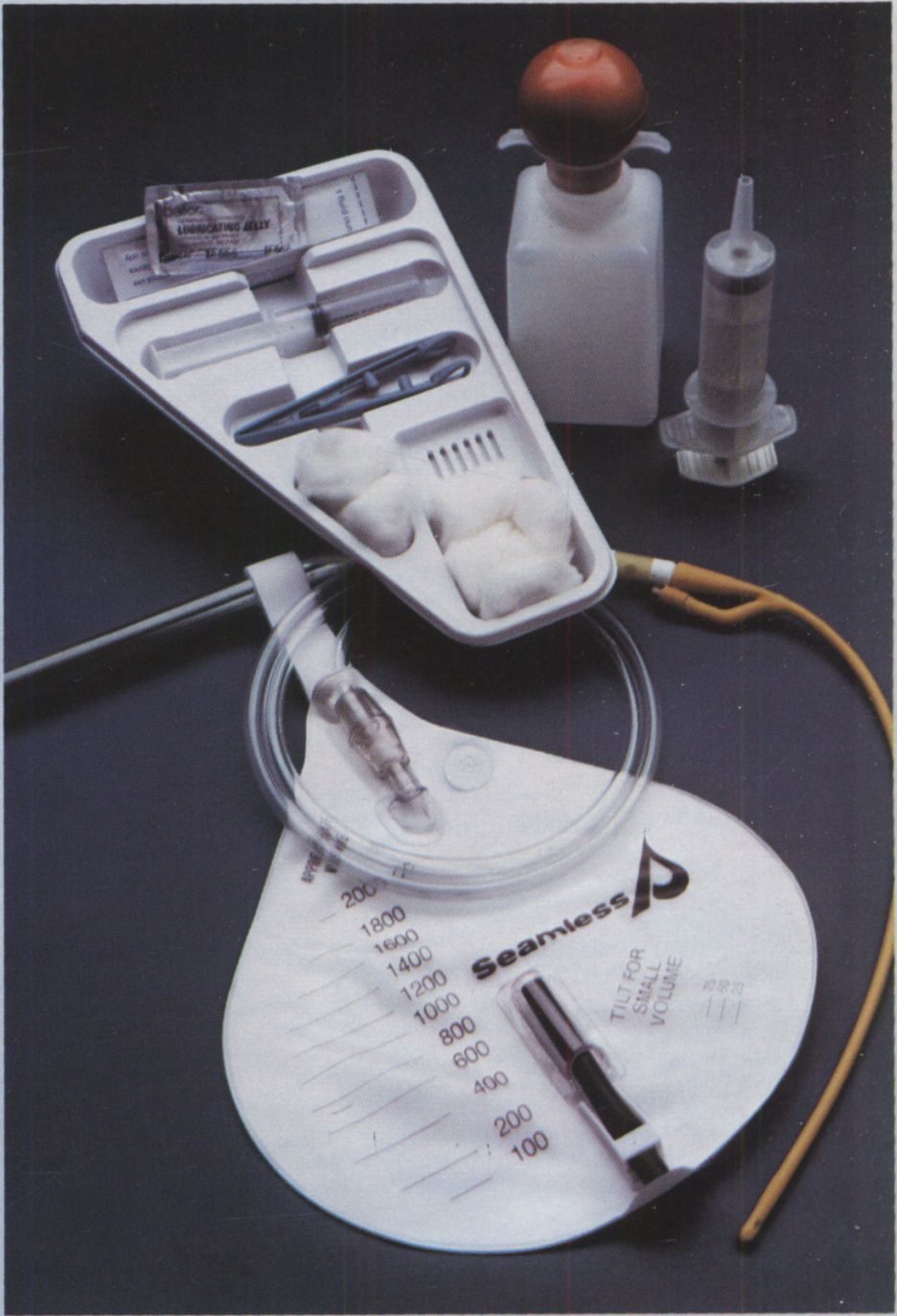

FREE Infection Control File

We don't think a drainage system has to be complex to be effective. But what do the investigators have to say? To find out, ask for our Clinical Papers Collection.... a complete selection of significant articles which evaluate uninary drainage systems... all in an easy-to-file binder. II's yours free just by calling 800-243-3030. 